\section{Six cases of autoimmune acquired coagulation factor VIII deficiency: Single center experience in Japan}

\author{
Akiko Konishi, Aya Nakaya, \\ Kazuyoshi Ishii, Shosaku Nomura \\ First Department of Internal \\ Medicine, Kansai Medical University, \\ Osaka, Japan
}

\begin{abstract}
We report six cases of autoimmune acquired coagulation factor VIII deficiency, which is a rare bleeding disorder. It is an autoimmune disease, however, there are various causes. We experienced cases with malignancy, co-exist with another autoimmune disease, pregnancy, and unknown epidemiology with repeated bleeding episode. All patients were controlled the acute bleeding phase and they have been under treatment with immunosuppression.
\end{abstract}

\section{Case Reports}

\section{Patients with malignancy}

Six individuals with Autoimmune Acquired Coagulation Factor Deficiency (AiFD) attended Kansai Medical University Hospital (Table 1). Three of them had histories of malignant disease. Patients 1 and 2 had histories of colon cancer more than three years before with no current evidence of active disease.

Patient 1 presented with a hematoma on the buttocks of rapid onset and was found to have a prolonged activated partial thromboplastin time (aPTT) (113.8 seconds). Factor VIII was under $1 \%$ and inhibitor against FVIII was $2070 \mathrm{BU} / \mathrm{mL}$. Then, she was diagnosed as having AiFD. She was given recombinant factor VIIa (rFVIIa) as acute treatment. Once control of the hematoma had been achieved, she was switched to prednisolone (PSL: $1 \mathrm{mg} / \mathrm{kg} /$ day) and cyclophosphamide (CSA: 100mg/day) as an inhibitor eradication therapy; however, this did not adequately control her aPTT and the level of inhibitor. Accordingly, rituximab was substituted. This therapy achieved good control that is currently being maintained with PSL.

Patient 2 presented with subcutaneous bleeding. aPTT was 82 seconds. Factor VIII was under $1 \%$ and inhibitor against FVIII was $1587 \mathrm{BU} / \mathrm{mL}$. He was diagnosed as having an AiFD. He was commenced on PSL and CSA, which achieved good control of his condition. The control was then maintained with PSL.

Patient 3 had breast cancer. She was found incidentally to have a prolonged aPTT (62.3 seconds); this was asymptomatic. She had $26 \%$ of factor VIII, although the amount of inhibitor was not determined. There was therefore insufficient evidence to diagnose AiFD. Nevertheless, she was started on PSL $(1 \mathrm{mg} / \mathrm{kg} /$ day $)$ on the basis of the clinical diagnosis. These three patients' coagulation disorders are still under control with maintenance PSL therapy at the time of writing.

\section{Patients with autoimmune disease}

Two of the patients who presented with AiFD had preexisting autoimmune diseases. Patient 4 had scleroderma that was well controlled with low dose of PSL (5-10 $\mathrm{mg}$ /day). She presented with subcutaneous bleeding of sudden onset. aPTT was 113.3 seconds. Factor VIII was under $1 \%$ and inhibitor against FVIII was $36.7 \mathrm{BU} / \mathrm{mL}$. She was negative for lupus anticoagulant. She was diagnosed as having AiFD. When the dose of PSL was increased (1 $\mathrm{mg} / \mathrm{kg} /$ day), her symptoms resolved. Patient 5 had a prior diagnosis of Rheumatoid Arthritis (RA), for which she was receiving no medication. She had recently been pregnant and was diagnosed in the third trimester as having placenta previa. She accordingly underwent cesarean section. Control of bleeding during the operation was good; however, she noticed hematuria a few days after the operation. She was found to have a prolonged aPTT (104.5 seconds). Factor VIII was under $1 \%$ and inhibitor against FVIII was $174 \mathrm{BU} / \mathrm{mL}$. She was negative for lupus anticoagulant. She was diagnosed as having AiFD. Her hematuria was slow to improve. She was therefore started on PSL (1 $\mathrm{mg} / \mathrm{kg} /$ day), which achieved good control of her symptoms.

\section{Patient with repeated episodes of bleeding}

Patient 6 had a long history of bleeding episodes, the first of which had occurred 22 years ago, at which time he had been diagnosed as having 'AiFD for factor IX' (supporting data unavailable). He had received PSL and CSA. He stopped attending for follow-up and 6 years later again presented with bleeding episodes. This time the diagnosis was 'AiFD for factor IX and VIII'. He received PSL pulse therapy and again stopped attending for follow-up.
Correspondence: Fumizono-cho, Moriguchi, Osaka 570-8507, Japan.

Tel.: +81-6-6992-1001 - Fax: +81-6-6992-1066 E-mail:nakaya1016@yahoo.co.jp

Key words: Autoimmune acquired coagulation factor VIII deficiency, malignancy, autoimmune disease, sudden bleeding

Conflict of interest: The authors declare no conflict of interest.

Funding: none.

Consent for publication: Informed consent has been obtained before publication.

Availability of data and materials: All data and materials are available within the main text.

Received for publication: 3 March 2020.

Accepted for publication: 30 November 2020 .

This work is licensed under a Creative Commons Attribution-NonCommercial 4.0 International License (CC BY-NC 4.0).

${ }^{(}{ }^{C}$ Copyright: the Author(s), 2020

Licensee PAGEPress, Italy

Hematology Reports 2020; 12:8525

doi:10.4081/hr.2020.8525

Three years later, he again presented with bleeding, for which he received rFVIIa and PSL pulse therapy. Eleven years later, he again presented with bleeding. On this occasion, his aPTT was prolonged (66.9 seconds), factor VIII was $24 \%$ and factor IX $17 \%$. The concentration of inhibitor of factor VIII was $0.7 \mathrm{BU} / \mathrm{mL}$ and of inhibitor of factor IX $1.0 \mathrm{BU} / \mathrm{mL}$. He was negative for lupus anticoagulant. Although the concentrations of inhibitor were low, his history of repeated bleeding prompted restarting treatment with PSL. His aPTT returned to within the normal range and factor VIII and IX activities increased in parallel with decreases in inhibitor concentrations.

\section{Discussion}

AiFD is a rare bleeding disorder, its incidence being $1.5 /$ million/year. $^{1}$ It is characterized by development of immunemediated antibodies to coagulation factors, resulting in bleeding episodes of sudden onset, especially in older individuals with no history of bleeding disorder. Although it is possible to create antibodies to any coagulation factor, the most commonly targeted coagulation factor is VIII, followed 
Table 1. Patients' characteristics.

\begin{tabular}{|c|c|c|c|c|c|c|c|c|c|}
\hline Case & Age & Sex & Past history & Symptom & $\begin{array}{l}\text { aPTT } \\
(\sec )\end{array}$ & $\begin{array}{l}\text { FVIII } \\
(\%)\end{array}$ & $\begin{array}{l}\text { Inhibitor } \\
\text { against FVIII } \\
(\mathrm{BU} / \mathrm{mL})\end{array}$ & $\begin{array}{l}\text { Treatment } \\
\text { treatment }\end{array}$ & Current \\
\hline 1 & 75 & Female & Colon cancer & Hematoma & 113.8 & $<1$ & 2070 & rFVIIa/PSL/CSA/Rituximab & PSL \\
\hline 2 & 80 & Male & Colon cancer & Subcutaneous bleeding & 82 & $<1$ & 1587 & PSL & $\mathrm{PSL} / \mathrm{CSA}$ \\
\hline 3 & 66 & Female & Breast cancer & No symptom & 62.3 & 26 & - & PSL & PSL \\
\hline 4 & 63 & Female & Scleroderma & Subcutaneous bleeding & 113.3 & $<1$ & 36.7 & PSL & PSL \\
\hline 5 & 34 & Female & RA/pregnancy/placenta previa & Hematuria & 104.5 & $<1$ & 174 & PSL & PSL \\
\hline 6 & 48 & Male & Prior bleeding episode & Hematoma & 66.9 & 24 & 0.7 & rFVIIa/PSL & PSL \\
\hline
\end{tabular}

RA: rheumatoid arthritis, aPTT: activated partial thromboplastin time, FVIII: factor VIII, rFVIIa: recombinant factor VIIa.

by factor V, XIII and von Willebrand Factor (vWF). ${ }^{2,3}$ In contrast to patients with congenital bleeding disorders, who commonly bleed into joints, most individuals with AiFD have subcutaneous bleeding, retroperitoneal hematomas, and bleeding into muscles or intestine. In more than half of patients with AiFD the condition develops spontaneously $(51.3 \%)$. Of the remainder, $13.1 \%$ have preexisting autoimmune disorders, $12.3 \%$ have had malignancy, $8.8 \%$ are postpartum, $4.1 \%$ are associated with infection and $3.7 \%$ are drugrelated. ${ }^{3}$ Treatment for acute bleeding is rFVIIa or activated prothrombin complex concentrate (aPCC). After achieving control of the acute bleeding, PSL with or without CSA is the preferred first-line therapy. If it is ineffective, rituximab, cyclosporine, or azathioprine may achieve the required immunosuppression. ${ }^{4,5}$

Three of our patients had histories of malignancy but had no evidence of tumor at the time of onset of AiFD. Therefore, we could not ascertain whether activity of malignant disease influences development of AiFD. Two of our patients had preexisting autoimmune diseases. Patient 4 was receiving treatment for scleroderma; however, Patient 5 was not receiving any treatment for her RA. The latter patient had recently been pregnant; thus, her AiFD may have been attributable more to her postpartum state than to her RA. Patient 6 had repeated episodes of bleeding for so long that we were unable to elicit all the details concerning his initial episode and diagnosis. At the beginning of the bleeding episode that prompted his presentation to our institution, his factor VIII inhibitor concentration was $0.7 \mathrm{BU} / \mathrm{mL}$, which is very low. However, his factor VIII activity was $24 \%$, indicating that neutralizing antibodies had attached to the antigens, creating immune complexes, thus lowering the measurable concentrations of factor VIII inhibitor. In such cases, it is possible that recovery of activity of factor VIII is a key indicator of a favorable response to treatment. However, the association of inhibitor against more than one factor is a very rare. Therefore, in this case, there must have been a possibility that it reflected laboratory interference.

Only two of our patients presented with severe acute bleeding. This was difficult to control with first-line treatment in Patient 1; therefore, rituximab was substituted.

\section{Conclusions}

It is difficult to diagnose AiFD because it is so rare that the possibility does not immediately come to mind and the specialized tests required for diagnosis may not be available in community hospitals. However, once the disease has been encountered, it is easy to keep it in mind, particularly because the symptoms and changes in coagulation data are so specific.

When a physician sees a patient with bleeding of sudden onset and no history of prior episodes, they should consider the possibility of AiFD and arrange the appropriate investigations.

\section{References}

1. Knoebl P, Marco P, Baudo F, et al. EACH2 Registry Contributors. Demographic and clinical data in acquired hemophilia A: results from the European Acquired Haemophilia Registry (EACH2). J Thromb Haemost 2012;10:622-31.

2. Kessler CM, Knöbl P. Acquired haemophilia: an overview for clinical practice. Eur J Haematol 2015;95:36-44.

3. Sborov DW, Rodgers GM. How I manage patients with acquired haemophilia A. Br J Haematol 2013;161: 157-65.

4. W Collins P, Chalmers E, Hart D, et al. Diagnosis and management of acquired coagulation inhibitors: a guideline from UKHCDO. Br J Haematol 2013;162: 758-73.

5. Kruse-Jarres R, Kempton CL, Baudo F, et al. Acquired hemophilia A: Updated review of evidence and treatment guidance. Am J Hematol 2017;92:695705. 